\title{
A heavily pretreated patient with recurrent clear cell adenocarcinoma of the ovary in whom carcinomatous peritonitis was controlled successfully by salvage therapy with gemcitabine
}

Shin-ichi Komiyama • Masaru Nakamura

Isao Murakami · Yoshiko Kuwabara · Takashi Kurahashi •

Kyoko Tanaka $\cdot$ Mikio Mikami

Published online: 5 December 2008

(C) Springer-Verlag 2008

Erratum to: Arch Gynecol Obstet (2008) 278:565-568

DOI 10.1007/s00404-007-0396-3

Unfortunately, first name of the corresponding author name was published incorrect in the online and print version of the article. Instead of "Shin Komiyama, it should read as "Shin-ichi Komiyama".

The online version of the original article can be found under doi:10.1007/s00404-007-0396-3.

S. Komiyama $(\square)$

Department of Obstetrics and Gynecology,

Fujita Health University School of Medicine,

Toyoake, 470-1192 Aichi, Japan

e-mail:komiyama@fujita-hu.ac.jp

S. Komiyama - M. Nakamura - I. Murakami · Y. Kuwabara ·

T. Kurahashi $\cdot$ K. Tanaka $\cdot$ M. Mikami

Department of Obstetrics and Gynecology,

National Saitama Hospital, Saitama, Japan

M. Mikami

Department of Obstetrics and Gynecology,

Tokai University School of Medicine, Kanagawa, Japan 